\title{
MIMO Channel Prediction Results on Outdoor Collected Data
}

\author{
Patrick Bidigare \\ Raytheon BBN Technologies Arlington, VA 22209 \\ bidigare@ieee.org \\ D. Richard Brown III \\ Worcester Polytechnic Institute Worcester, MA 01609 \\ drb@wpi.edu
}

\author{
Shawn Kraut \\ MIT Lincoln Laboratories, Lexington, MA 02421 \\ kraut@11.mit.edu \\ Upamanyu Madhow \\ University of California, Santa Barbara CA 93106 \\ madhow@ece.ucsb.edu
}

\begin{abstract}
In this paper we analyze the performance of an autoregressive MIMO channel predictor on outdoor data collected with both vehicular and pedestrian transmitter motion. The metric of performance considered is aggregate beamforming gain obtained using dominant eigenmode MIMO communications when the transmit beamforming vector is chosen based on the channel prediction. We show that while the prediction range obtained on this real data is somewhat less than earlier published results based on simulated data, we are still able to obtain $2 \mathrm{X}$ to $3 \mathrm{X}$ greater feedback latency tolerance than without using these predictive techniques.
\end{abstract}

\section{INTRODUCTION}

Communications channel prediction is an essential technique for utilizing adaptive transmission schemes in scenarios where the channel varies rapidly due to motion and multipath. The adaptive transmission scheme of particular interest to the authors is informed transmitter MIMO across a distributed transmit array such as that shown in Fig. 1 . Coherent transmission (beamforming) across a distributed network of radios causes the expected received power to grow quadratically with the number of transmitting radios. This feature can enable long distance communications using a network of small, low-power radios [1-7].

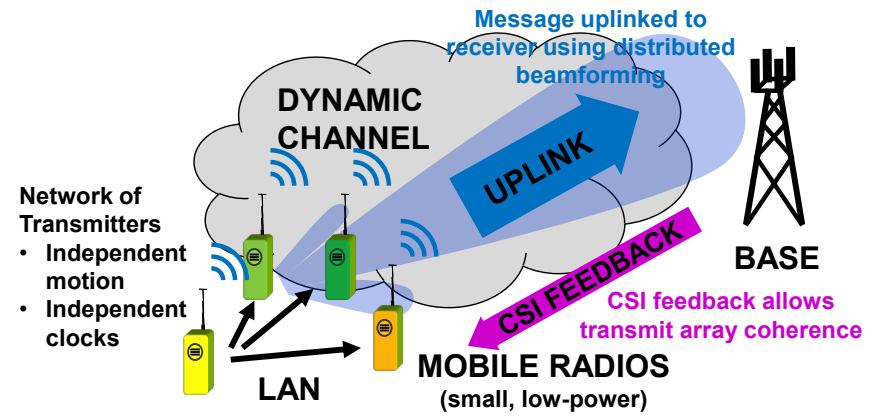

Fig. 1 Receiver-coordinated distributed transmit beamforming allows an adhoc network of transmitters to achieve longer communication ranges.

If it were possible to know the current channel state information at the transmit array, the size of the distributed array could be chosen to achieve arbitrarily long uplink ranges, but unfortunately estimation of the MIMO channel coefficients does not scale with the size of the array. As communications range increases, the per-channel SNR decreases and more integration time must be used during channel sounding to achieve an accurate CSI estimate. This effect is shown in Fig. 2 for a feedback-based system that interleaves incoherent channel sounding slots and coherent uplink beamforming slots. This CSI estimation requirement ultimately limits the communications range that can be achieved using feedbackbased informed transmit techniques in dynamic channel environments [8-10]. The minimum latency of the CSI feedback is half the channel sounding interval plus the time needed to calculate the CSI from the sounding samples and feed this back over the downlink channel.

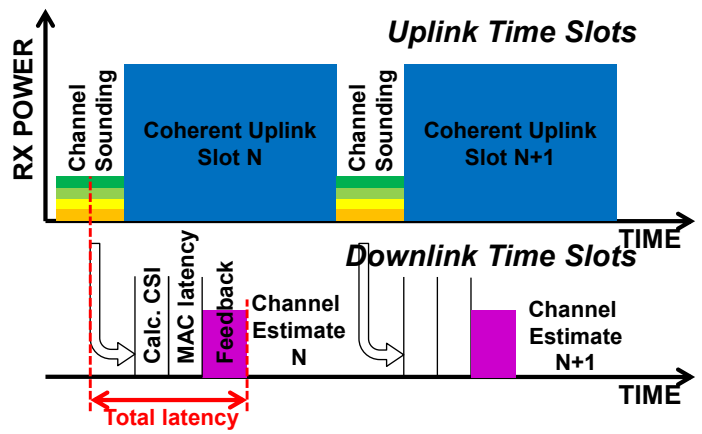

Fig. 2 Feedback latency is fundamentally limited by the duration of the channel sounding which must increase as per-channel SNR decreases at long ranges.

This minimum latency can easily be greater than the interval over which the channel can be modeled as stationary. In these situations, channel prediction techniques can increase the amount of latency that can be tolerated.

Dozens papers have addressed the problem of multipath channel prediction (see [11] for survey). The communications channel is generally modeled as a superposition of paths, each with its own complex amplitude and Doppler frequency which are either constant or change very slowly (Fig. 3 ).

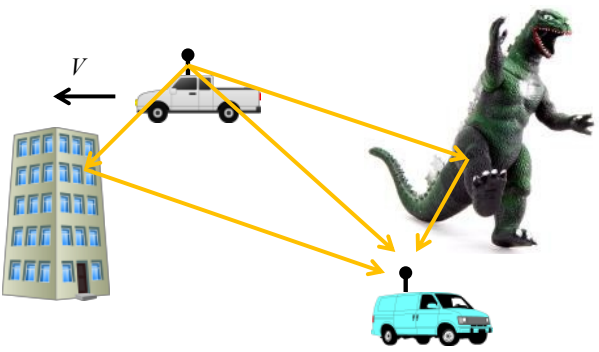


Fig. 3 Superposition of scattering paths leads to sum-of-sinusoids channel evolution model.

The majority of papers use a sum-of-sinusoids (1) or autoregressive process (2) to model the channel.

$$
\begin{gathered}
\text { flat fading channel } \\
\text { path amplitude } \\
x(t)=\sum_{t=1}^{L} \gamma_{l} \exp \left(2 \pi f_{l} t\right) \quad \begin{array}{c}
\text { max } \\
f_{\max }=V / \lambda
\end{array} \\
x[n]:=x\left(n / f_{s}\right) \quad \begin{array}{c}
\text { Channel sounding } \\
\text { frequency } \\
f_{s}>2 f_{\max }
\end{array} \\
x[n]=\sum_{p=1}^{P} a_{p} x[n-p]+e[n]
\end{gathered}
$$

The best SISO channel predictors have an accurate envelope prediction range (time) of $\lambda / V$ to $1.5 \lambda / V$ in rich multipath scattering environments [12], where $\lambda$ is the wavelength and $V$ is the vehicle velocity. A CRLB analysis [13] suggests that MIMO channels can be predicted over considerably longer intervals than SISO channels by exploiting the common Doppler shifts of different propagation paths and the spatial structure imposed by the transmit and receive antenna array manifolds.

All published prediction results, to the authors' knowledge, have used simulated channels or idealized move-stop-move indoor channel measurements. The contribution of this work is the analysis of data from an outdoor MIMO collection campaign

\section{DAtA Collection \& PROCESSING}

\section{A. Collection}

In May 2012 a series of data collections was performed by MIT Lincoln Laboratory at their campus on Hanscom Air Force Base in Lexington, MA. The collections utilized a 4 channel transmitter and an 8 channel receiver. In all collections, the receive antennas mounted on the top of a van (Fig. 4 right) which was stationary throughout the duration of the collection. Three different configurations of the four transmit antennas were used (Fig. 4 left):

\section{A static linear array of transmit antennas}

2. Transmit antennas mounted to the top of a truck, circling a parking structure at $\sim 10 \mathrm{mph}$.

3. Transmit antennas hand-held while people are bobbing and changing orientation
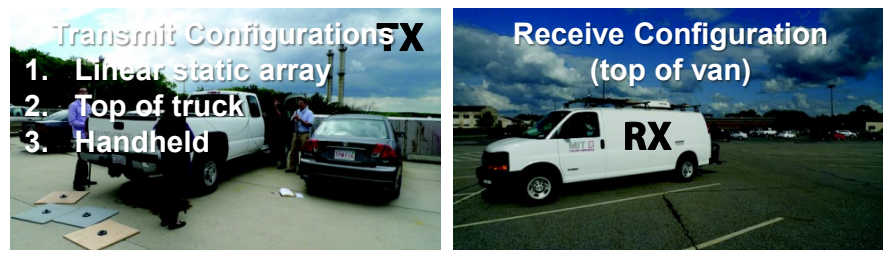

Fig. 4 MIMO collection configuration.

In each configuration, independent $\mathrm{PN}$ sequences from each of the four transmit antennas and simultaneously sampled from the eight receive antennas. In this collection, a common oscillator was used for the transmitters as well as the receivers and both oscillators were disciplined to GPS, thus the channel dynamics are entirely due to transmitter or environmental motion. The parameters of the collection are summarized in TABLE I.

TABLE I. EXPERIMENT PARAMETERS

\begin{tabular}{lcc} 
Parameter & \multicolumn{2}{c}{ Value } \\
\hline \# Antennas & TX: $4 \quad$ RX: 8 \\
Height & TX: $16 \mathrm{~m} \quad$ RX: $2 \mathrm{~m}$ \\
Frequency & $375 \mathrm{MHz}$ \\
Bandwidth & $800 \mathrm{kHz}$ \\
Range & $1.5 \mathrm{~km}$ \\
Duration & $\sim 1 \mathrm{~s}$
\end{tabular}

\section{B. Channel Estimation}

In order to apply channel prediction techniques to the MIMO channels, it was first necessary to estimate these channels from the eight channels of receive samples knowing the four PN sequences transmitted. Each of the $4 \times 8=32$ SISO channels was modeled as a 20-tap FIR filter and the LMS algorithm was used to adaptively estimate the channel coefficients to minimize the mean square error (Fig. 5 ).

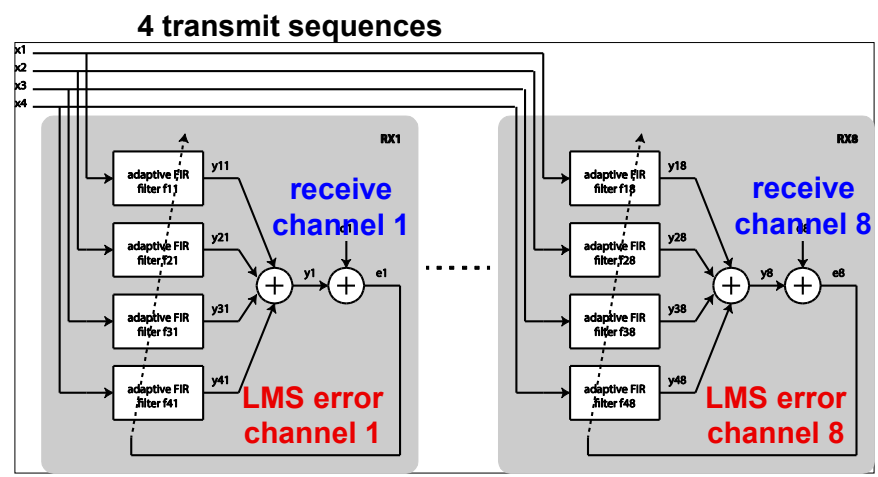

Fig. 5 LMS provides a recursive solution for the minimum mean square (MMSE) channel estimate

As illustrates, the LMS residual error is low, suggesting that the technique is generating good channel estimates.
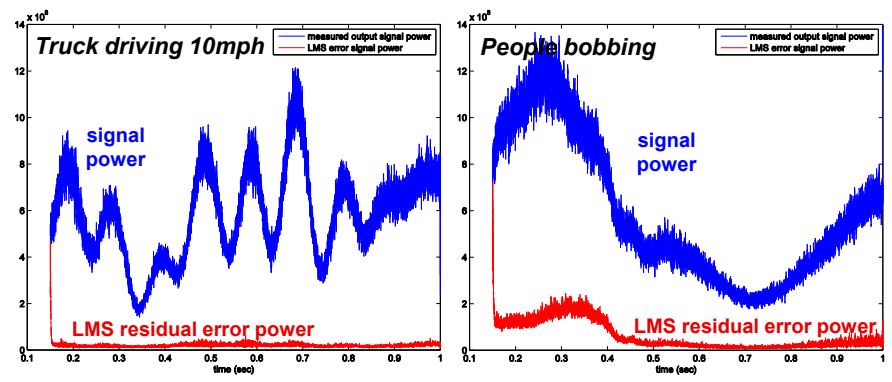

Fig. 6 Average LMS error residual power is typically $1-5 \%$ of the measured output signal power.

After processing, the resulting channel coefficients form a fourdimensional dataset indexed by transmitter, receiver, delay tap and time (in $1 \mathrm{~ms}$ increments). Fig. 7 shows the MIMO channel for three of the four transmitters, all 8 receivers and the middle 14 taps for one particular point in time. Here phase is mapped to hue and magnitude is mapped to intensity. 


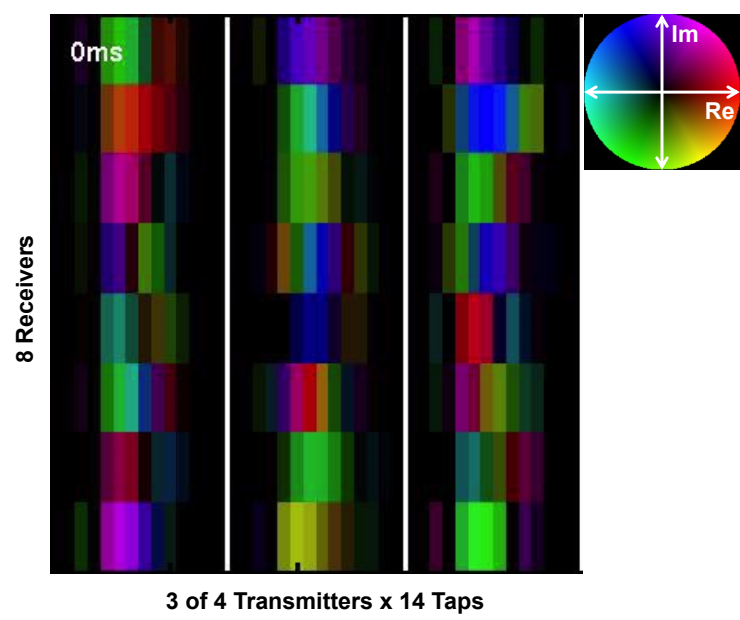

Fig. 7 Frequency selective MIMO channel at one snapshot in time.

\section{Channel Prediction}

For the purposes of this analysis, we treat each tap of each transmit-receive pair as an independent $\mathrm{AR}(2)$ process and fit the coefficients to the evolution of the estimated channel. Let $\mathrm{x}[\mathrm{n}]$ denote the channel coefficient corresponding to a particular (tap, TX channel, RX channel) triplet, then we model

$$
x[n]=a_{1} x[n-1]+a_{2} x[n-2]+u[n]
$$

where $u[n]$ is assumed to be a white noise process sequence. Each tap, transmitter, receiver triplet gets its own set of complex AR coefficients. We utilized the Burg method to estimate these coefficients. The Burg method guarantees a stable AR process model.

Once the AR process coefficients for a particular channel are estimated, they can be used to predict the value of the channel in the future. We can rewrite (3) as

$$
\underbrace{\left[\begin{array}{c}
x[n+1] \\
x[n]
\end{array}\right]}_{\mathbf{x}[n+1]}=\underbrace{\left[\begin{array}{cc}
a_{1} & a_{2} \\
1 & 0
\end{array}\right]}_{\mathbf{A}} \underbrace{\left[\begin{array}{c}
x[n] \\
x[n-1]
\end{array}\right]}_{\mathbf{x}[n-1]}+\left[\begin{array}{c}
u[n] \\
0
\end{array}\right]
$$

Given our assumption on the AR process noise, the MMSE pstep predictor is simply

$$
\hat{\mathbf{x}}_{a r}[n+p]=\mathbf{A}^{p} \mathbf{x}[n],
$$

where $x[n]$ corresponds to the known channel state at time $n$ as generated by the LMS algorithm above. It is natural to compare the performance of this predictor against the "trivial" stationary predictor given by

$$
\hat{\mathbf{x}}_{t r i v}[n+p]=\mathbf{x}[n],
$$

Fig. 8 shows the performance of the AR predictor against the true channel when using $60 \mathrm{~ms}$ of training and predicting ahead $40 \mathrm{~ms}$.

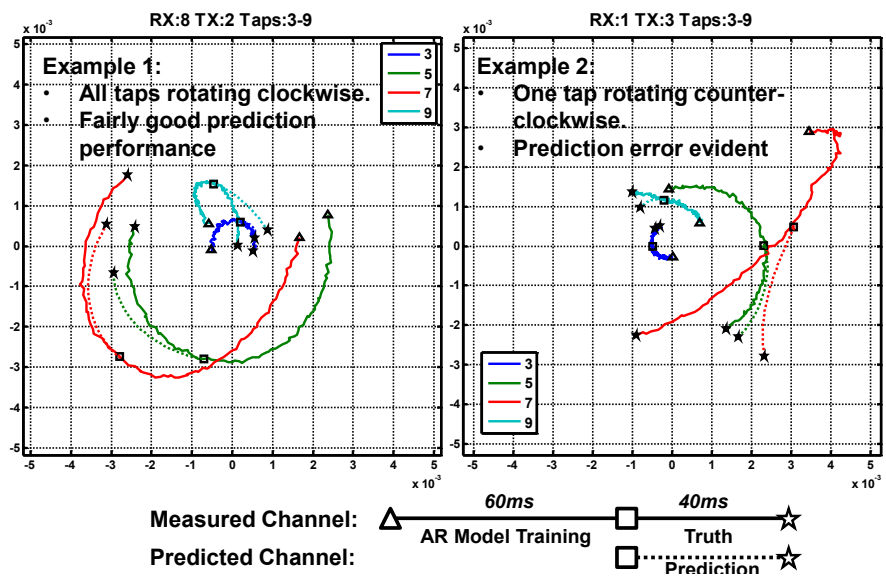

Fig. 8 Two examples of channel prediction performance against collected data for transmitters on a $10 \mathrm{mph}$ truck.

\section{PERFormanCE}

\section{A. Performance Metric}

Most previous work on channel prediction has directly characterized the channel tracking accuracy; however this is only indirectly related to communications performance. The motivating application for this work is feedback-based dominant eigenmode MIMO communications, which uses beamforming on transmit and receive to maximize the aggregate SNR of the SISO channel formed. In this context, achieved beamforming is the appropriate performance metric for comparing the performance of a channel prediction scheme.

Fig. 9 illustrates dominant eigenmode MIMO communications where transmit and receive beamforming vectors are chosen to maximize the SNR of the resulting SISO channel.

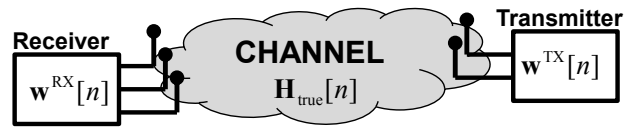

Fig. 9 Dominant eigenmode MIMO communications

When the true channel matrix $\mathbf{H}_{\text {true }}[n]$ of a frequency-flat MIMO channel is known, the optimal beamforming vectors are obtained by first performing a singular value decomposition of the channel

$$
\mathbf{H}_{\text {true }}[n]=\mathbf{U}[n] \mathbf{S}[n] \mathbf{V}[n]^{H}
$$

and then selecting the receive and transmit beamforming vectors to be the left and right singular vectors corresponding to the largest (dominant) singular value

$$
\mathbf{w}_{\text {opt }}^{\mathrm{RX}}[n]=\mathbf{u}_{1}[n], \mathbf{w}_{\text {opt }}^{\mathrm{TX}}[n]=\mathbf{v}_{1}[n]
$$

in which case the maximum beamforming gain of $S_{11}[n]$ is achieved.

The true channel matrix is not available at the transmitter primarily because of the feedback latencies discussed above and the transmitter must rely on an estimate $\hat{\mathbf{H}}[n]$ of the channel provided by the receiver. This estimate can be obtained using channel prediction techniques. The optimal 
transmit beamforming vector given this channel estimate is the dominant right singular vector of the estimate

$$
\hat{\mathbf{w}}^{\mathrm{TX}}[n]=\hat{\mathbf{v}}_{1}[n] \text { where } \hat{\mathbf{H}}[n]=\hat{\mathbf{U}}[n] \hat{\mathbf{S}}[n] \hat{\mathbf{V}}[n]^{H}
$$

We note, however, that while feedback latency affects the fidelity of the channel estimate available to the transmitter, the receiver knows the current true channel state (up to channel estimation error which we ignore here) and can use this knowledge, along with the transmitters chosen beamforming vector, in selecting its receive beamforming vector. In a whitenoise interference environment, the receive beamforming vector that maximizes beamforming gain is the matched filter

$$
\hat{\mathbf{w}}^{\mathrm{RX}}[n]=\frac{\mathbf{H}_{\text {ture }}[n] \hat{\mathbf{v}}_{1}[n]}{\left\|\mathbf{H}_{\text {true }}[n] \hat{\mathbf{v}}_{1}[n]\right\|}
$$

The beamforming loss, relative to omniscient knowledge of the channel can be calculated for a particular channel estimator as

$$
\eta_{d B}[n]=10 \log _{10}\left(\frac{\mathbf{H}_{\text {true }}[n] \hat{\mathbf{v}}_{1}[n]}{\left\|\mathbf{H}_{\text {true }}[n] \hat{\mathbf{v}}_{1}[n]\right\| S_{11}[n]}\right)
$$

These equations generalize to a frequency selective formulation.

\section{B. Evaluation Methodology}

Channel prediction performance was computed as follows:

- STEP 1: The AR model discussed above was used to independently predict each component (tap, transmitter, receiver) of the MIMO channel for prediction ranges of $10 \mathrm{~ms}, 30 \mathrm{~ms}, 50 \mathrm{~ms}$ and $100 \mathrm{~ms}$.

- STEP 2: Beamforming gain was computed for (a) the true channel (optimal beamforming), (b) the AR-predicted channal and (c) the trivial channel predictor obtained by assuming a stationary channel.

Fig. 10 and Fig. 11 show, respectively, the beamforming performance of the $\operatorname{AR}(2)$ channel predictor and the trivial channel predictor at 3 different prediction intervals. The AR(2) predictor clearly out-performs the stationary predictor for vehicular motion.
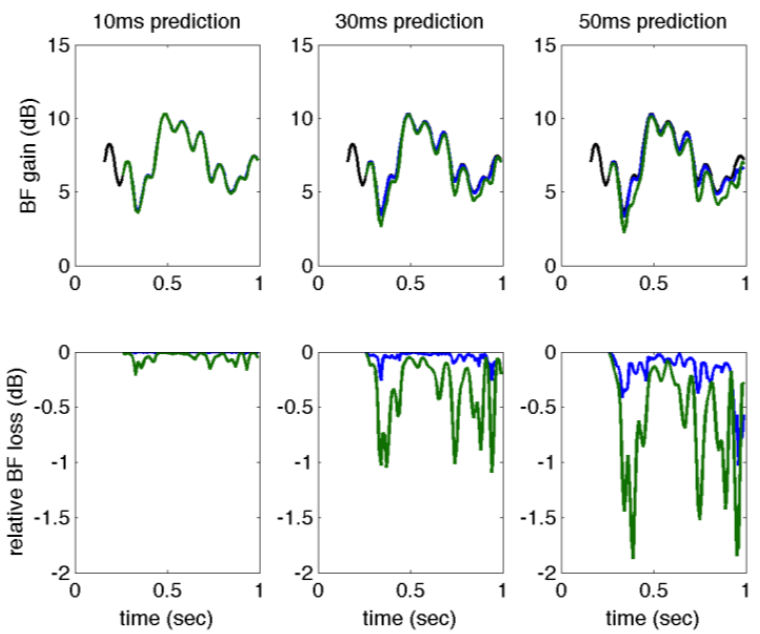

Fig. 10 Beamforming gain (top) and relative beamforming loss (bottom) for the $10 \mathrm{mph}$ truck collection. Three different prediction intervals show performance of omniscient channel knowledge (black), the AR(2) predictor (blue) and the trivial stationary estimate (green).
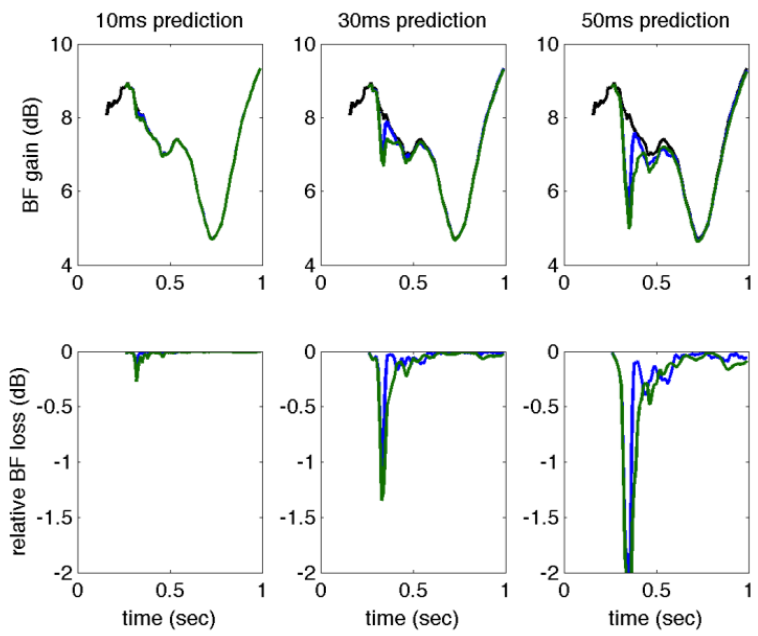

Fig. 11 Beamforming gain (top) and relative beamforming loss (bottom) for the "people bobbing" collection. Three different prediction intervals show performance of omniscient channel knowledge (black), the AR(2) predictor (blue) and the trivial stationary estimate (green).

\section{Beamforming Loss Distributions}

Fig. 12 shows the cumulative distribution (over time) of the channel predictors.
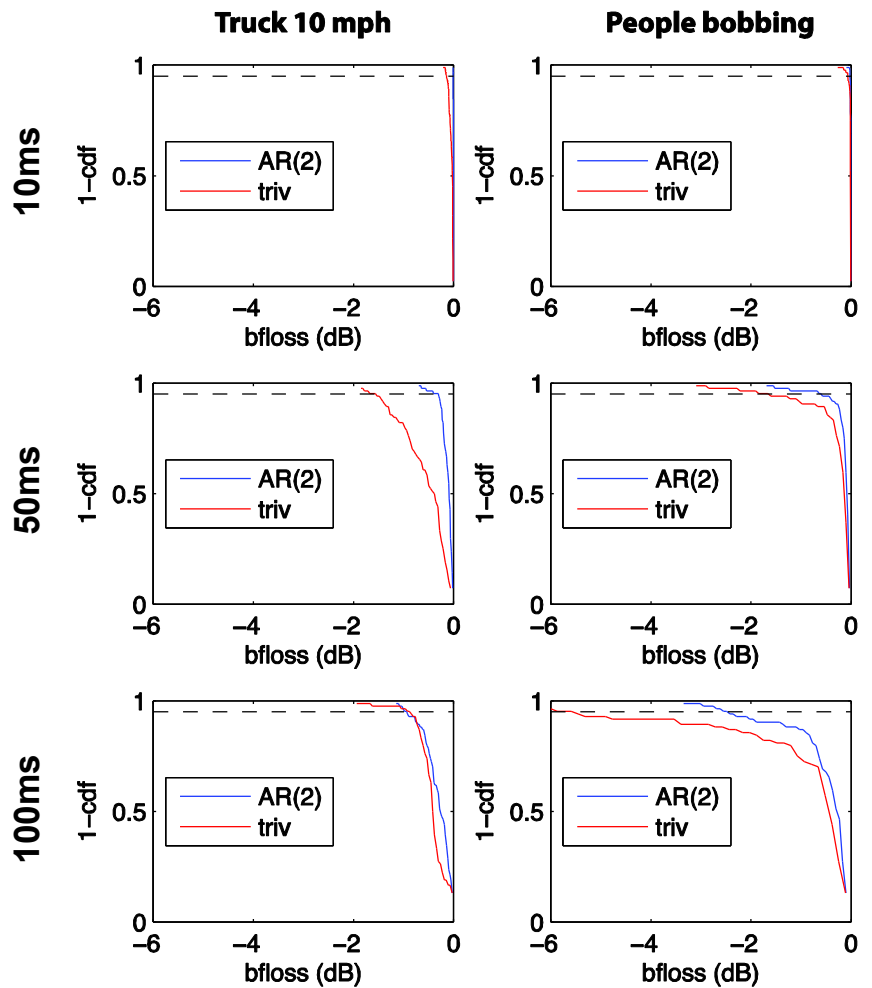

Fig. 12 Cumulative distributions of beamforming loss (relative to omniscient channel knowledge) for the AR(2) (blue) and trivial (red) predictors at three different prediction ranges. 
We use the dashed $95 \%$ point to quantify the beamforming loss vs. prediction range for the channel predictors.

\section{CONClusions AND Future WORK}

TABLE II. summarizes the prediction performance of the $\mathrm{AR}$ and trivial channel predictors against the vehicular and pedestrian data collections as well as the expected performance of AR SISO channel predictors documented in the literature.

TABLE II. RESULTS SUMMARY

\begin{tabular}{lcc} 
& Vehicular & Pedestrian \\
\hline Wavelength & & $0.8 \mathrm{~m}$ \\
Maximum velocity & $4.5 \mathrm{~m} / \mathrm{s}$ & $\sim 1 \mathrm{~m} / \mathrm{s}$ \\
Prediction range & $180 \mathrm{~ms}$ to $280 \mathrm{~ms}$ & $800 \mathrm{~ms}$ to $1200 \mathrm{~ms}$ \\
$(\lambda / \mathrm{V}$ to $1.5 \lambda / \mathrm{V})$ & & \\
AR predictor range & $\sim 100 \mathrm{~ms}$ & $\sim 60 \mathrm{~ms}$ \\
Trivial predictor range & $\sim 30 \mathrm{~ms}$ & $\sim 30 \mathrm{~ms}$
\end{tabular}

As defined, the $\mathrm{AR}(2)$ predictor offers a channel prediction range of about $0.5 \lambda / \mathrm{V}$ for a vehicular motion channel and only a $0.1 \lambda / \mathrm{V}$ prediction range for a channel with pedestrian motion dynamics. In contrast, simulation results from the literature suggest that prediction ranges of $1.0 \lambda / \mathrm{V}$ to $1.5 \lambda / \mathrm{V}$ are possible using AR channel predictors. The performance mismatch is likely because the assumption of nearly-constant path amplitudes and Doppler frequency shifts is less applicable to these datasets. Nevertheless, the AR(2) predictor supports $2 \mathrm{X}$ (pedestrian) or $3 \mathrm{X}$ (vehicular) greater latencies than the trivial predictor.

Future work in this area may attempt to incorporate the spatial and delay structures of the MIMO channel into the AR model as suggested in [13]. This approach is challenging because it introduces non-linearities into the channel model.

\section{REFERENCES}

[1] D. R. Brown, P. Bidigare, and U. Madhow, "Receiver coordinated distributed transmit beamforming with kinematic tracking," in IEEE International Conference on Acoustics, Speech, and Signal Processing (ICASSP 2012), Kyoto, Japan, March 2012.
[2] R. Mudumbai, G. Barriac, and U. Madhow, "On the feasibility of distributed beamforming in wireless networks," IEEE Trans. on Wireless Communications, vol. 6, no. 5, pp. 1754-1763, May 2007

[3] R. Mudumbai, D.R. Brown III, U. Madhow, and H.V. Poor, "Distributed transmit beamforming: Challenges and recent progress," IEEE Communications Magazine, vol. 47, no. 2, pp. 102-110, February 2009.

[4] Mudumbai, Raghu; Madhow, Upamanyu; Brown, Rick; Bidigare, Patrick; , "DSP-centric algorithms for distributed transmit beamforming," Signals, Systems and Computers (ASILOMAR), 2011 Conference Record of the Forty Fifth Asilomar Conference on, vol., no., pp.93-98, 6-9 Nov. 2011

[5] M. M. U. Rahman, H. E. Baidoo-Williams, S. Dasgupta, and R. Mudumbai, "Fully wireless implementation of distributed beamforming on a software-defined radio platform," in the 11th ACM/IEEE Conference on Information Processing in Sensor Networks (IPSN), 2012.

[6] F. Quitin, U. Madhow, M. Rahman, and R. Mudumbai, "Demonstrating distributed transmit beamforming with software-defined radios," in World of Wireless, Mobile and Multimedia Networks (WoWMoM), 2012 IEEE International Symposium on a, pp. 1 -3, june 2012.

[7] P. Bidigare et. al., "Implementation and demonstration of receivercoordinated distributed transmit beamforming across an ad-hoc radio network", Signals, Systems and Computers (ASILOMAR), 2012 Conference Record of the Forty Sixth Asilomar Conference on, pp. 22226, 4-7 Nov. 2012

[8] B. Hassibi and B. M. Hochwald, "How much training is neededin multiple-antenna wireless links?" IEEE Trans. on Information Theory, vol. 49, pp. 951-963, Apr. 2003.

[9] W. Santipach and M.L. Honig, "Optimzation of training and feedback overhead for beamforming over block fading channels", IEEE Trans. on Information Theory, vol. 56, pp. 6103-6115, Dec. 2010.

[10] A. Margetts, E. Torkildson, R. Bartlett and S. Kraut, "Optimal Training and Data Power Allocation for Distributed Transmit Beamforming", Signals, Systems and Computers (ASILOMAR), 2013 Conference Record of the Forth Seventh Asilomar Conference on, to appear.

[11] A. Duel-Hallen, "Fading Channel Prediction for Mobile Radio Adaptive Transmission Systems", Proceedings of the IEEE, Vol 95, No. 12, Dec 2007, pp. 2299-2313.

[12] S. Semmelrodt and R. Kettenbach, "Investigation of different fading forecast schemes for flat fading radio channels", Vehicular Technology Conference, 2003, VTC 2003-Fall. 2003 IEEE $58^{\text {th }}$ vol 1, pp. 149-153 69 Oct 2003.

[13] T. Svantesson and A.L. Swindlehurst, "A performance bound for prediction of MIMO channels," Signal Processing, IEEE Transactions on, vol.54, no.2, pp.520,529, Feb. 2006 